\title{
Spontaneous pneumomediastinum in patients with severe acute respiratory syndrome
}

\author{
C.M. Chu*, Y.Y. Leung*, J.Y.H. Hui", I.F.N. Hung ${ }^{+}$, V.L. Chan*, W.S. Leung*, K.I. Law", C.S. Chan", \\ K.S. Chan*, K.Y. Yuen ${ }^{+}$
}

Spontaneous pneumomediastinum in patients with severe acute respiratory syndrome. C.M. Chu, Y.Y. Leung, J.Y.H. Hui, I.F.N. Hung, V.L. Chan, W.S. Leung, K.I. Law, C.S. Chan, K.S. Chan, K.Y. Yuen. (C) ERS Journals Ltd 2004.

ABSTRACT: Spontaneous pneumomediastinum (SP) unrelated to assisted ventilation is a newly recognised complication of severe acute respiratory syndrome (SARS). The objective of the present study was to examine the incidence, risk factors and the outcomes of SP in a cohort of SARS victims from a community outbreak.

Data were retrieved from a prospectively collected database of virologically confirmed SARS patients. One hundred and twelve cases were analysable, with 13 patients developing SP $(11.6 \%)$ at a mean \pm SD of $19.6 \pm 4.6$ days from symptom onset.

Peak lactate dehydrogenase level was associated with the development of SP. SP was associated with increased intubation and a trend towards death. Drainage was required in five cases. For patients who survived, the SP and/or the associated pneumothoraces took a median of 28 days (interquartile range: 15-45 days) to resolve completely.

In conclusion, spontaneous pneumomediastinum appeared to be a frequent complication of severe acute respiratory syndrome. Further research is needed to investigate its pathogenesis.

Eur Respir J 2004; 23: 802-804.
*Dept of Medicine, ${ }^{\#}$ Intensive Care Unit, and "Dept of Radiology, United Christian Hospital, and ${ }^{+}$Dept of Microbiology, Queen Mary Hospital, The University of Hong Kong, Hong Kong SAR, China.

Correspondence: C.M. Chu, Division of Respiratory Medicine, Dept of Medicine, United Christian Hospital, Hong Kong SAR, China.

Fax: 85223472325

E-mail: nncmchu@netvigator.com

Keywords: Complication, coronavirus, pneumomediastinum, pneumothorax, severe acute respiratory syndrome

Received: August 222003

Accepted after revision: January 222004
Severe acute respiratory syndrome (SARS) has been documented to be caused by a novel coronavirus (SARS-CoV) [1-3], which satisfied the Koch's postulations for causation [4, 5]. At the time of writing, the numbers of probable SARS cases has reached 8,422 globally [6]. Two previous studies have noted the occurrence of spontaneous pneumomediastinum (SP) occasionally in patients with SARS, unrelated to assisted ventilation [7, 8]. However, SP in SARS has not been systematically studied. In the present study the incidence, risk factors and the implications of the development of SP in SARS sufferers were examined.

\section{Methods}

The present study examined SP in SARS patients by retrospective analysis of a prospectively collected SARS database. Patients included in the study were consecutive SARS patients admitted to the United Christian Hospital (Hong Kong, SAR, China) during a community outbreak from March 24 to April 28, 2003. Patients met a modified WHO definition of SARS, which included fever $\left(\geqslant 38^{\circ} \mathrm{C}\right)$, cough or shortness of breath, new pulmonary infiltrates on radiological examination, in the absence of an alternative diagnosis, together with virological documentation of SARS-CoV infection (paired serology and/or positive RT-PCR for SARS-CoV from clinical specimens). All patients were treated with a standard protocol of broad spectrum antibiotics, ribavirin and a tailing regimen of corticosteroids [9]. The clinical, haematological, biochemical, radiological and virological findings were prospectively entered into a preset database, according to previous publications [1,7].

Chest radiographs were taken at intervals of 1 to 3 days, depending on clinical need. SP was defined as the presence of gas in the mediastinum, occurring before assisted ventilation. For equivocal cases, high resolution computed tomography (HRCT) of the thorax would be utilised to detect this complication. All chest radiographs and HRCT were interpreted by thoracic radiologists.

Results were expressed as mean \pm SD unless otherwise specified. Univariate analyses of potential factors associated with the occurrence of SP were performed with the unpaired t-test, Mann-Whitney U-test or Fisher's exact test where appropriate. A two-tailed p-value of $<0.05$ was considered statistically significant.

\section{Results}

There were 123 virologically confirmed SARS patients admitted to hospital during the study period. Altogether, there were 24 patients with pneumomediastinum: 13 had SP and 11 developed pneumomediastinum with or without pneumothorax after the commencement of assisted ventilation. The baseline characteristics were not significantly different between those with SP and those with ventilatorassociated barotraumas (VAB) (table 1). However, the cumulative dose of methylprednisolone received by the SP group prior to the onset of pneumomediastinum was significantly lower than that received by the VAB group (median: 2.0 versus $3.0 \mathrm{~g}$, respectively, $\mathrm{p}=0.003$ ).

Eleven cases of VAB were excluded from further analysis. This left 112 cases for analysis, including 13 cases complicated by SP. The mean age of the 112 cases was $38.8 \pm 12.7$ yrs. There were 46 males $(41.1 \%)$ and 66 females $(58.9 \%)$. The initial chest radiographs appeared normal in $28(25 \%)$ 
Table 1.-Comparisons of initial clinical, radiological and laboratory features between severe acute respiratory syndrome (SARS) patients with spontaneous pneumomediastinum (SP) and ventilator-associated barotraumas (VAB)

\begin{tabular}{lccl}
\hline \multicolumn{1}{c}{ Parameters } & SP & VAB & p-value \\
\hline Subjects n & 13 & 11 & \\
Fever & $13(100)$ & $11(100)$ & 1.0 \\
Chills & $8(61.5)$ & $6(54.5)$ & 1.0 \\
Rigors & $6(46.2)$ & $6(54.5)$ & 1.0 \\
Myalgia & $8(61.5)$ & $10(90.9)$ & 0.166 \\
Cough & $8(61.5)$ & $7(63.6)$ & 1.0 \\
Dyspnoea & $9(69.2)$ & $5(45.8)$ & 0.408 \\
Diarrhoea & $7(53.8)$ & $7(63.6)$ & 0.697 \\
Ever-smoker & $0(0)$ & $0(0)$ & 1.0 \\
Normal chest radiograph & $2(15.4)$ & $4(36.4)$ & 0.357 \\
Multilobar involvement & $3(23.1)$ & $1(9.1)$ & 0.596 \\
Haemoglobin $\mathrm{g} \cdot \mathrm{dL}^{-1}$ & $13.5(12.7-14.9)$ & $14.1(13.5-14.7)$ & 0.531 \\
Lymphocyte $\times 10^{9} \cdot \mathrm{L}^{-1}$ & $0.8(0.6-1.4)$ & $0.7(0.6-0.8)$ & 0.424 \\
Platelet $\times 10^{9} \cdot \mathrm{L}^{-1}$ & $191(144-232)$ & $162(146-197)$ & 0.569 \\
Initial LDH $\mathrm{IU} \cdot \mathrm{L}^{-1}$ & $412(357-505)$ & $344(293-523)$ & 0.207 \\
\hline
\end{tabular}

Data are presented as $\mathrm{n}(\%)$ or median (interquartile range) unless otherwise stated. SP: spontaneous pneumomediastinum; VAB: ventilatorassociated barotraumas.

patients, who had HRCT findings compatible with SARS. Multilobar involvement was evident on presentation in 21 $(18.8 \%)$ cases.

Thirteen patients developed SP (11.6\%) during the course of SARS, at a mean of $19.6 \pm 4.6$ days from symptom onset. Of these 13 cases, one had concomitant subcutaneous emphysema, three had simultaneous spontaneous bilateral pneumothoraces, and two had subsequent progression into subcutaneous emphysema and bilateral pneumothoraces. One patient had pneumomediastinum, bilateral pneumothoraces and subcutaneous emphysema (fig. 1). Bilateral tube thoracostomies were necessary in the five cases complicated by bilateral pneumothoraces, of whom one case received chemical pleurodesis; the rest of the SP was managed conservatively. No patient in this series had isolated pneumothorax and all pneumothoraces occurred concomitantly with, or were preceded by, SP.

Univariate analysis showed that only peak serum lactate dehydrogenase (LDH) was associated with the development of SP $(\mathrm{p}=0.001)$. Other factors, including viral load in the nasopharynx, cumulative dose of corticosteroids, initial radiographical findings and other laboratory parameters were not associated with the development of SP (table 2).

Of the 13 patients with SP, five were intubated and two of the intubated patients died; two additional patients with SP died without intubation on surrogates' requests, giving a total of four deaths in SP patients. Patients who developed SP had a statistically higher chance of intubation $(p=0.015)$ and showed more of a trend towards death $(\mathrm{p}=0.057)$ (table 1$)$. For patients who survived, the SP and/or the associated pneumothoraces took a median of 28 days (interquartile range: 15-45 days) to resolve completely.

\section{Discussion}

SP developed in $11.6 \%$ of the present study SARS cohort at 19.6 \pm 4.6 days from symptom onset. High peak LDH level was associated with its development. Development of SP was associated with significant intubation rate and mortality. In survivors, resolution of SP was slow.

Pneumomediastinum is the presence of extra-alveolar air in the mediastinum. Free air leaks from ruptured alveoli,

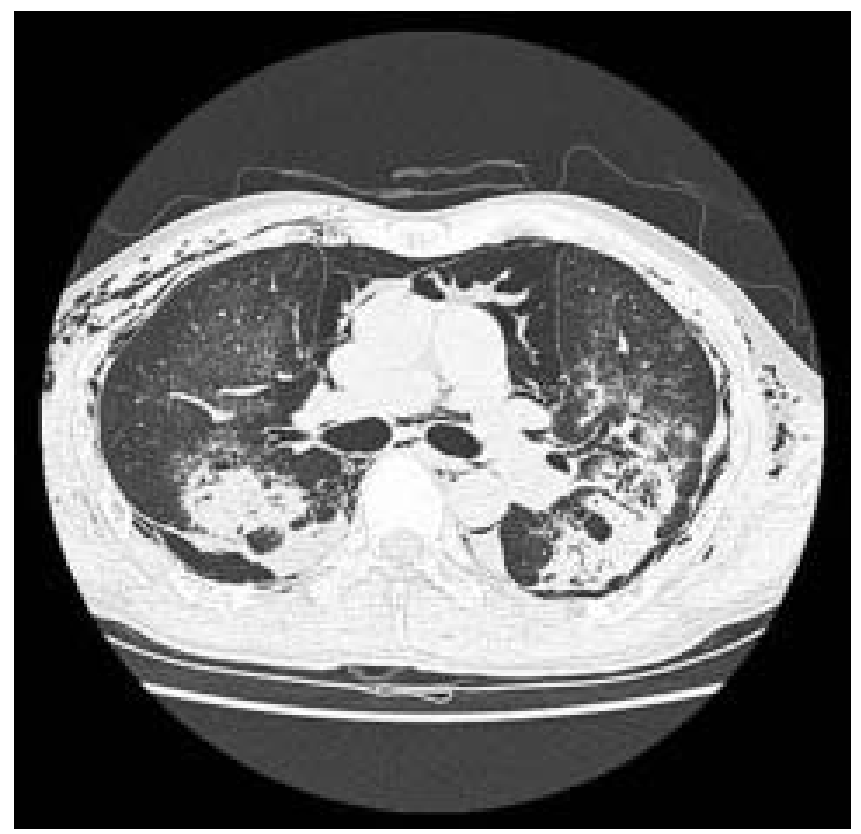

Fig. 1.-High-resolution computed tomography (HRCT) thorax of a patient with severe acute respiratory syndrome (SARS) complicated by spontaneous pneumomediastinum, subcutaneous emphysema and rims of bilateral pneumothoraces.

dissecting along the bronchovascular sheaths towards the mediastinum [10]. The incidence of SP was reported to be one in 32,896 in the general population [11]. In immunocompetent hosts with pulmonary infections, air leak as a complication is uncommon, but it has been reported in staphylococcal pneumonia [12] and fungal pneumonia [13]. It has also been reported to complicate interstitial pneumonitis secondary to systemic lupus erythematosus [14] and bronchiolitis obliterans organising pneumonia [15]. In HIV infection, SP is reported in association with Pneumocystis carinii pneumonia in $9.5 \%$ and with tuberculosis in $6.8 \%$ of cases [16]. SP is also reported to complicate cytomegalovirus pneumonitis in patients with haematological malignancies [17] and idiopathic pneumonia syndrome after bone marrow transplantation [18].

It was interesting to note that isolated pneumothorax, without a preceding or concomitant pneumomediastinum, was not observed in the current series of patients. One possible explanation is that the current study examined the patients regularly by chest radiographs and/or HRCT. This might have given the authors an opportunity to witness a sequence of events that took place in these severely lungdamaged patients. The current authors postulate that alveolar rupture may occur in SARS as a result of severe diffuse alveolar damage [19] and cause interstitial emphysema. Air may then dissect along the bronchovascular sheaths into the mediastinum to manifest as SP initially, which may further progress to pneumothoraces and subcutaneous emphysema in some patients.

VAB in the current study was, by definition, not spontaneous and, therefore, could not be classified as SP. However, their clinical, laboratory and radiological features did not seem to be fundamentally different from those of SP. The higher dose of methylprednisolone received in patients with VAB versus those with SP was probably an indicator of the need for more intensive treatment in sicker patients. It is possible that both SP and VAP in SARS share similar pathogenic mechanisms, although mechanical ventilation may further aggravate barotraumas and air leak.

High peak LDH level, which might signify cellular damage, 
Table 2. - Comparisons of potential risk factors and the outcomes of severe acute respiratory syndrome (SARS) patients with and without spontaneous pneumomediastinum (SP)

\begin{tabular}{|c|c|c|c|}
\hline Parameter & No SP & SP & p-value \\
\hline Subjects $n$ & 99 & 13 & \\
\hline Mean \pm SD age yrs & $39.4 \pm 12.9$ & $34.1 \pm 9.6$ & 0.153 \\
\hline Male & $38(38.4)$ & $8(61.55)$ & 0.138 \\
\hline Smoking status: nonsmoker, exsmoker, current smoker & $\begin{array}{c}92,4,3 \\
(93,4,3)\end{array}$ & $\begin{array}{c}13,0,0 \\
(100,0,0)\end{array}$ & 0.612 \\
\hline Active comorbidity & $13(13.1)$ & $3(23.1)$ & 0.394 \\
\hline Apparently normal chest radiograph on admission & $23(23.2)$ & $5(38.5)$ & 0.305 \\
\hline Multilobar involvement on initial chest radiograph & $20(20.2)$ & $1(7.7)$ & 0.456 \\
\hline Haemoglobin $\mathrm{g} \cdot \mathrm{dL}^{-1}$ & $13.5 \pm 1.6$ & $14.2 \pm 0.8$ & 0.052 \\
\hline Initial total peripheral WBC count $\times 10^{9} \cdot \mathrm{L}^{-1}$ & $6.3 \pm 2.1$ & $7.5 \pm 2.7$ & 0.055 \\
\hline Initial lymphocyte count $\times 10^{9} \cdot \mathrm{L}^{-1}$ & $0.9 \pm 0.5$ & $0.8 \pm 0.3$ & 0.458 \\
\hline Initial platelet count $\times 10^{9} \cdot \mathrm{L}^{-1}$ & $170 \pm 49$ & $172 \pm 37$ & 0.910 \\
\hline Initial urea $\mathrm{mmol} \cdot \mathrm{L}^{-1}$ & $4.1 \pm 1.2$ & $4.4 \pm 1.1$ & 0.475 \\
\hline Initial creatinine $\mu \mathrm{mol} \cdot \mathrm{L}^{-1}$ & $85.5 \pm 16.0$ & $90.7 \pm 14.3$ & 0.266 \\
\hline Initial LDH IU $\cdot \mathrm{L}^{-1}$ & $379(313-449)$ & $344(296-518)$ & 0.663 \\
\hline peak LDH IU $\cdot \mathrm{L}^{-1}$ & $583(471-746)$ & $863(702-1485)$ & 0.001 \\
\hline Cumulative methylprednisolone dose $\mathrm{g}$ & $2.3 \pm 1.1$ & $2.4 \pm 0.5$ & 0.741 \\
\hline Viral load in NP specimen on presentation $\log _{10}$ RNA copies $\cdot \mathrm{mL}^{-1}$ & $0(0-5.0)$ & $0(0-4.6)$ & 0.493 \\
\hline Viral load in NP specimen peak $\log _{10}$ RNA copies $\cdot \mathrm{mL}^{-1}$ & $0(0-5.3)$ & $4.5(0-6.6)$ & 0.169 \\
\hline Intubation & $10(10.1)$ & $5(38.5)$ & 0.015 \\
\hline Death & $10(10.1)$ & $4(30.8)$ & 0.057 \\
\hline
\end{tabular}

Data are presented as mean $\pm \mathrm{SD}, \mathrm{n}(\%)$ or median (interquartile range) unless otherwise stated; WBC: white blood cell; NP: nasopharyngeal; LDH: lactate dehydrogenase.

was associated with the development of SP. In contrast, viral load was not related to the development of SP, suggesting processes other than viral-induced cytolysis might be important in the pathogenesis of alveolar damage (e.g. immunopathological damage). In this connection, it is interesting to note that the onset of SP (mean: 19 days) occurs after the phase of viral replication (day 10), at a time when immunopathological damage is thought to be important [7].

In conclusion, spontaneous pneumomediastinum appeared to be a characteristic and frequent complication of severe acute respiratory syndrome. It portends a poor outcome. Further research is needed to investigate the pathogenesis of spontaneous pneumomediastinum in severe acute respiratory syndrome.

\section{References}

1. Peiris JS, Lai ST, Poon LL, et al. Coronavirus as a possible cause of severe acute respiratory syndrome. Lancet 2003; 361: 1319-1325.

2. Ksiazek TG, Erdman D, Goldsmith CS, et al. A novel coronavirus associated with severe acute respiratory syndrome. N Engl J Med 2003; 348: 1953-1966.

3. Drosten C, Gunther S, Preiser W, et al. Identification of a novel coronavirus in patients with severe acute respiratory syndrome. N Engl J Med 2003; 348: 1967-1976.

4. Fouchier RA, Kuiken T, Schutten M, et al. Aetiology: Koch's postulates fulfilled for SARS virus. Nature 2003; 423 : 240.

5. Kuiken T, Fouchier RA, Schutten M, et al. Newly discovered coronavirus as the primary cause of severe acute respiratory syndrome. Lancet 2003; 362: 263-270.

6. World Health Organization. Summary table of SARS cases by country, 1 November 2002-7 August 2003. www.who.int/ csr/sars/country/en/country2003_08_15.pdf. Date last updated: August 15, 2003. Date last accessed: August 19, 2003.

7. Peiris JS, Chu CM, Cheng VC, et al. Clinical progression and viral load in a community outbreak of coronavirus-associated
SARS pneumonia: a prospective study. Lancet 2003; 361: 1767-1772.

8. Hui JYH, Cho DHY, Yang MKW, et al. Severe acute respiratory syndrome: spectrum of high resolution CT findings and temporal progression of the disease. $\mathrm{Am}$ $J$ Roentgen 2003; 181: 1525-1538.

9. So LK, Lau AC, Yam LY, et al. Development of a standard treatment protocol for severe acute respiratory syndrome. Lancet 2003; 361: 1615-1617.

10. Macklin CC. Transport of air along sheaths of pulmonic blood vessels from alveoli to mediastinum: clinical implications. Arch Intern Med 1939; 64: 913-926.

11. Bodey GP. Medical mediastinal emphysema. Ann Intern Med 1961; 54: 46-56.

12. Yates SP, Morcos SK. Delayed tension pneumothorax complicating staphylococcal pneumonia. Postgrad Med J 1988; 64: 796-798.

13. Ödev K, Çaliskan Ü, Emlik D, et al. Pneumomediastinum and pneumopericardium due to intracavitary aspergilloma: an unusual complication of fungal pneumonia. Pediatr Radiol 2002; 32: 143-145.

14. Masuda A, Tsushima $\mathrm{T}$, Shizume $\mathrm{K}$, et al. Recurrent pneumothoraces and mediastinal emphysema in systemic lupus erythematosus. J Rheumatol 1990; 17: 544-548.

15. Kofteridis D, Bouros DE, Vamvakas LN, et al. Pneumothorax complicating fatal bronchiolitis obliterans organizing pneumonia. Respiration 1999; 66: 266-268.

16. Tumbarello M, Tacconelli E, Pirronti T, et al. Pneumothorax in HIV-infected patients: role of Pneumocystis carinii pneumonia and pulmonary tuberculosis. Eur Respir J 1997; 10: $1332-1335$.

17. Olliff JFC, Williams MP. Radiological appearances of cytomegalovirus infection. Clin Radiol 1989; 40: 463-467.

18. Kudoh T, Suzuki N, Oda T, et al. Pneumomediastinum, subcutaneous emphysema, and pulmonary fibrosis in patient with idiopathic pneumonia syndrome after bone marrow transplantation. Pediatr Hematol Oncol 2000; 17: 113-117.

19. Nicholls JM, Poon LL, Lee KC, et al. Lung pathology of fatal severe acute respiratory syndrome. Lancet 2003; 361: $1773-1778$. 